PLANT SCIENCE

\section{Evolutionary flexibility in flooding response circuitry in angiosperms}

\author{
Mauricio A. Reynoso ${ }^{1 *} \dagger$, Kaisa Kajala ${ }^{2,3,4 *}$, Marko Bajic ${ }^{5,6 *}$, Donnelly A. West ${ }^{2 *}$, \\ Germain Pauluzzi $^{1 *}$, Andrew I. Yao ${ }^{2,3}$, Kathryn Hatch ${ }^{5}$, Kristina Zumstein ${ }^{2}$, \\ Margaret Woodhouse ${ }^{2}$, Joel Rodriguez-Medina ${ }^{2,3}$, Neelima Sinha $^{2} \neq$, \\ Siobhan M. Brady ${ }^{2,3} \ddagger$, Roger B. Deal ${ }^{5} \neq$, Julia Bailey-Serres ${ }^{1,4} \ddagger$
}

Flooding due to extreme weather threatens crops and ecosystems. To understand variation in gene regulatory networks activated by submergence, we conducted a high-resolution analysis of chromatin accessibility and gene expression at three scales of transcript control in four angiosperms, ranging from a dryland-adapted wild species to a wetland crop. The data define a cohort of conserved submergenceactivated genes with signatures of overlapping cis regulation by four transcription factor families. Syntenic genes are more highly expressed than nonsyntenic genes, yet both can have the cis motifs and chromatin accessibility associated with submergence up-regulation. Whereas the flexible circuitry spans the eudicot-monocot divide, the frequency of specific cis motifs, extent of chromatin accessibility, and degree of submergence activation are more prevalent in the wetland crop and may have adaptive importance.

C limate change has increased the frequency and intensity of floods that affect agricultural productivity. Of major crops, only rice [Oryza sativa $(O s)]$ is resilient to waterlogging of roots and submergence of aerial tissue, because of adaptation to a semiaquatic habitat. Other angiosperms experience intermittent flooding and are not adapted to these conditions. Submergence triggers signaling in plant cells as a consequence of entrapment of the gaseous hormone ethylene and depletion of available oxygen (hypoxia), leading to inefficient anaerobic metabolism and energy starvation (1). To understand the variation in response to submergence, we studied rice as a representative monocot and flood-resilient species, the legume Medicago truncatula (Mt), and two Solanum species, domesticated tomato [Solanum lycopersicum $\mathrm{S}(\mathrm{Sl})$ cultivar M82] and its dryland-adapted wild relative Solanum pennellii $(S p)$ (Fig. 1A). Roots are the first responders to flooding, and we thus monitored the early response of seedling apical root tips to complete seedling submergence. By monitoring the sentinel response gene family ALCOHOL DEHYDROGENASE (ADH),

\footnotetext{
${ }^{1}$ Center for Plant Cell Biology, Botany and Plant Sciences Department, University of California, Riverside, CA, USA. ${ }^{2}$ Department of Plant Biology, Division of Biological Sciences, University of California, Davis, CA, USA ${ }^{3}$ Genome Center, University of California, Davis, CA , USA. ${ }^{4}$ Institute of Environmental Biology, Utrecht University, $3584 \mathrm{CH}$ Utrecht, Netherlands. ${ }^{5}$ Department of Biology, Emory University, Atlanta, GA, USA. ${ }^{6}$ Graduate Program in Genetics and Molecular Biology, Emory University, Atlanta, GA, USA.

*These authors contributed equally to this work.

†Present address: Instituto de Biotecnología y Biología Molecular, FCE-UNLP CCT-CONICET, 1900 La Plata, Argentina.

‡Corresponding author. Email: roger.deal@emory.edu (R.B.D.); nrsinha@ucdavis.edu (N.S.); sbrady@ucdavis.edu (S.M.B.); serres@ucr.edu (J.B.-S.)
}

required for anaerobic production of adenosine $5^{\prime}$-triphosphate (1) (Fig. 1B), we identified 2 hours, the midpoint of maximal up-regulation, as a physiologically relevant time to compare initiation of the submergence response across species.

To conserve energy under hypoxia, stressinduced mRNAs are preferentially translated over transcripts associated with development in the model Arabidopsis thaliana (2-4). We therefore considered both transcriptional and posttranscriptional regulation under submergence across the species surveyed. To do so, we deployed Isolation of Nuclei TAgged in specific Cell Types (INTACT) (5) and Translating Ribosome Affinity Purification (TRAP) (6), using constitutive promoters. INTACT was used to profile chromatin accessibility by ATAC (assay for transposaseaccessible chromatin)-sequencing (ATAC-seq) (7) and to measure the abundance of nuclear RNA (nRNA). TRAP was used to monitor ribosomeassociated polyadenylated mRNA (TRAP RNA) and to evaluate the position of individual ribosomes along transcripts (Ribo-seq) (8) (Fig. 1C and figs. S1 and S2). We also profiled total polyadenylated mRNA (polyA RNA). Multidimensional scaling analysis confirmed the reproducibility and distinctness of each of the RNA subpopulations and their changes after submergence (fig. S3 and data S1 and S2).

Flood-adapted rice displayed the greatest plasticity in terms of the number of differentially up- and down-regulated transcripts (Fig. 1D, fig. S4, and data S3). Cultured hairy roots (Sl-HRs) were used as a contrast to intact roots of tomato $(S l)$ plants and were more responsive. The clustering of modulated RNAs resolved variation in regulation in all four species (Fig. 1D and figs. S5 to S9). Rice gene regulation was coordinated across scales (except in clusters 7 and 8, in which transcripts were enriched or depleted in the nucleus). In $M t$ and tomato, regulation of gene activity was more evident in the ribosome-associated RNAs, whereas in the dryland-adapted $S p$, regulation was evident as nRNA enrichment or depletion.

Selection likely acts on species-specific traits and adaptation to specific environments that are largely regulated by a common set of gene families. The root meristem is frequently oxygen deprived because of high metabolic activity and periodic soil inundation; therefore, its capacity to transiently up-regulate anaerobic metabolism might be expected in all species. Yet, rice may have evolved a higher proportion of gene family members that are regulated by submergence than flooding-sensitive species did. We leveraged gene families (9) to investigate conservation in submergence-responsive genes of the four species, focusing on the shared families (6685) plus those conserved between the two Solanum species (3301) (Fig. 1E and data S4). Tabulation of the submergence-responsive gene family members of each species identified families with at least one member differentially controlled in any of the RNA populations evaluated (Fig. 1F, fig. S10, and data S5). This uncovered a set of 68 submergenceup-regulated families (SURFs: 249 genes in $O s$, 121 in $M t, 137$ in $S l, 181$ in $S l$-HR, and 92 in $S p$ ). The 68 SURFs include 17 of the 49 ubiquitously hypoxia-responsive genes of Arabidopsis seedlings (6), demonstrating evolutionary conservation of gene families activated by submergence and hypoxia (data S5).

The 68 SURFs include 1 to 13 up-regulated genes per family, leading us to investigate whether similar proportions of these families are elevated in each species (fig. S11 and data S6). Consistent with overall numbers, rice had the highest and $S p$ had the lowest proportion of up-regulated genes per family. The restrained response of wild tomato was evident from the 412 Solanumspecific gene families that were up-regulated in tomato but not in $S p$. This motivated exploration of the aerial tissue (shoot apex) response in the Solanum species, which uncovered more gene families and family members up-regulated in shoots of wild tomato than those of domesticated tomato (fig. S12 and data S7). The shoot response of $S p$ showed greater overlap with Arabidopsis shoot-specific hypoxia-responsive genes (10). Distinctions between the two Solanum species included genes involved in cell elongation and auxin signaling, which predominated in $S p$.

We reasoned that dynamics in chromatin accessibility and transcriptional activation may be coordinated and conserved for SURF members across species. ATAC-seq exposed open chromatin regions of rice and $M t$ primarily within $1 \mathrm{~kb}$ upstream of the transcription start site (TSS) and downstream of the polyadenylation (pA) site of genes (Fig. 2A and data S8). By contrast, Solanum roots showed a majority of intergenic ATAC-seq reads (fig. S13). The rice and $M t$ transposase hypersensitive sites (THS) (11) uncovered a preference for opening of chromatin in response to submergence (Fig. 2B and fig. S13), with increases in 3497 and 7501 THSs, respectively. Highly submergence-up-regulated genes had elevated 
A

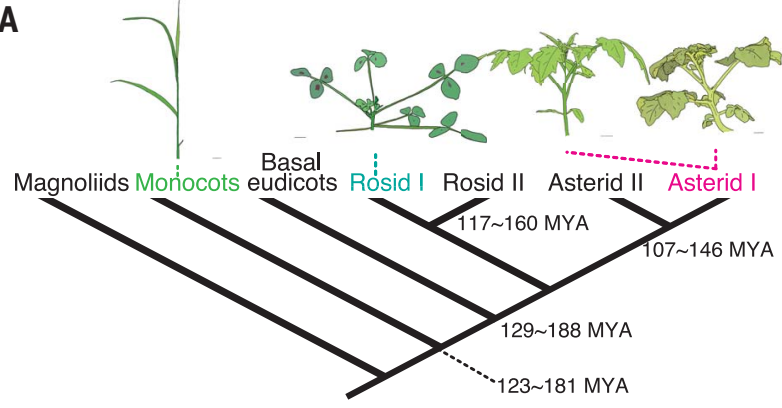

B

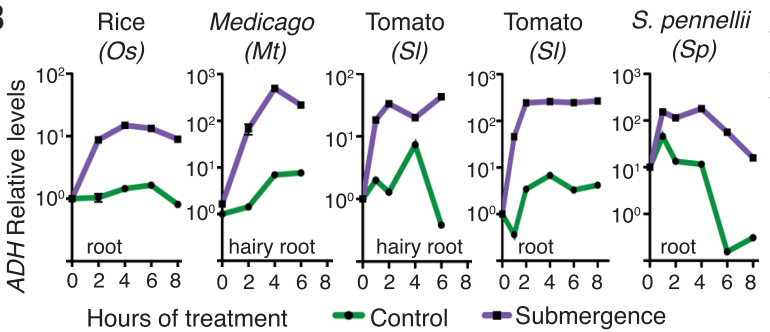

C

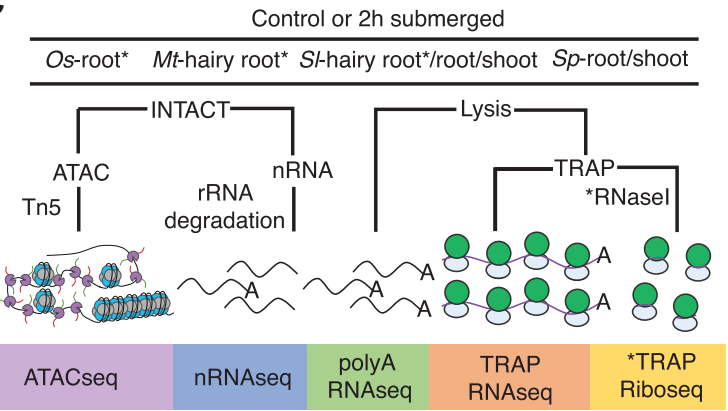

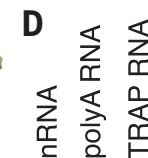
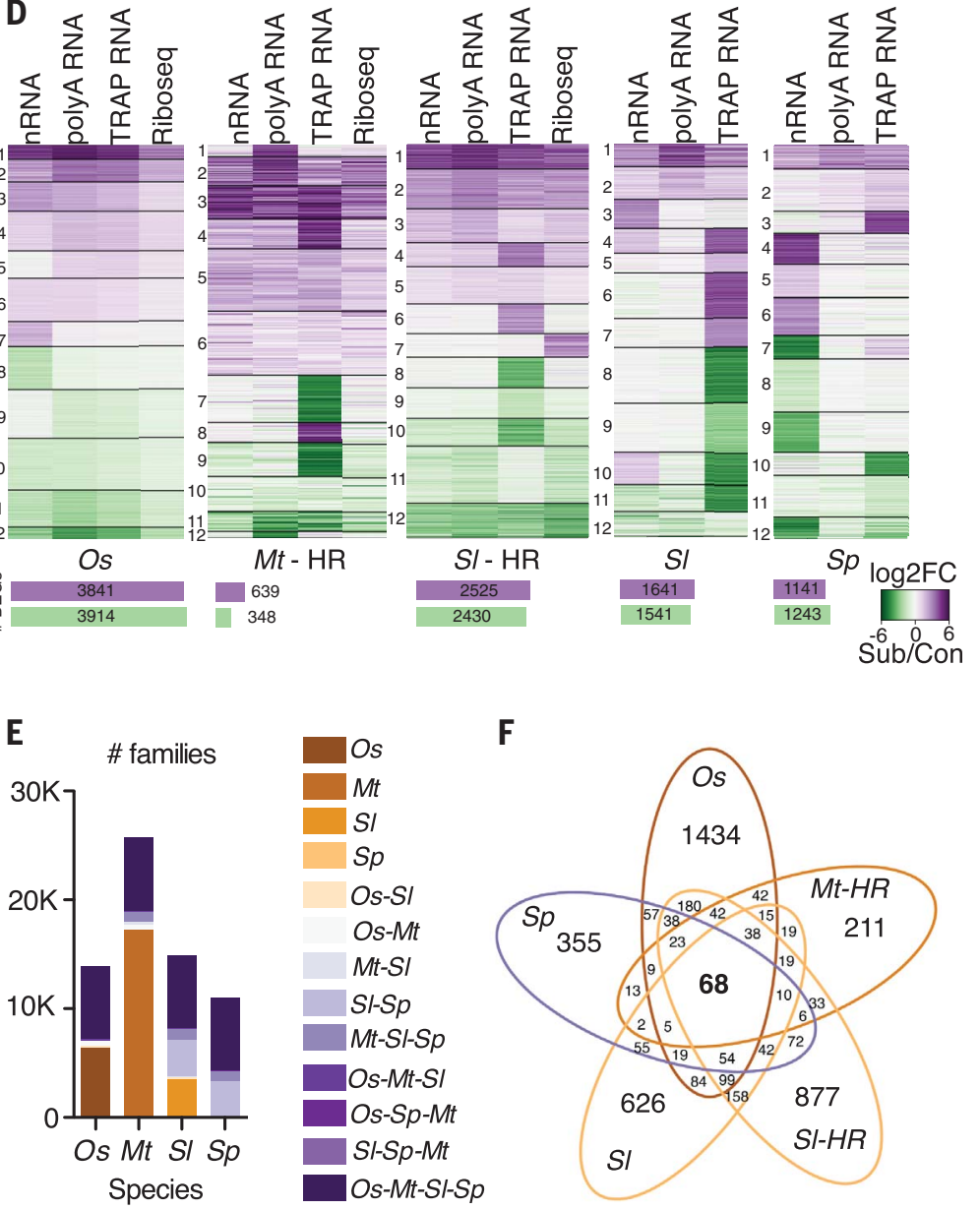

$\mathbf{F}$

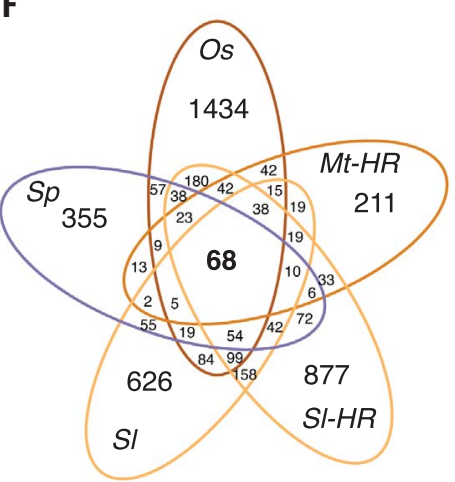

Fig. 1. Multitier evaluation of gene activity in four angiosperms identifies highly conserved submergence-up-regulated genes.

(A) Relatedness of target species (19). MYA, million years ago. (B) ADH transcript levels of submerged seedlings. (C) Overview of experimental strategy. rRNA, ribosomal RNA. (D) Cluster analysis heatmap of $\log _{2}$ fold change [FC; submergence (Sub) versus control (Con) RNA] of differentially expressed genes [DEGs; $\left|\log _{2} \mathrm{FC}\right|>1$ and adjusted $P($ Padj $)<0.01$ ].

(Bottom) Bars indicate number of up or down DEGs after submergence $\mid \log _{2}$ $\mathrm{FC} \mid>1$ and $P$ adj $<0.05$. (E) Gene families per species and their overlap.

(F) Conserved SURFs and species-specific up-regulated family numbers. accessibility $5^{\prime}$ of their TSS and $3^{\prime}$ of their pA sites (Fig. 2C and figs. S5, S6, and S14), demonstrating that nucleosome depletion accompanies activation of transcript production under submergence. Down-regulated genes had lower chromatin accessibility overall, particularly in rice (Fig. $2 \mathrm{C}$ and figs. S5, S6, and S14).

We exploited the ATAC-seq data to explore conservation in gene regulatory circuitry. A pipeline was developed to identify transcription factor (TF) binding site motif enrichment in promoters and their THS regions of the upregulated SURFs (Fig. 2D). Four significantly enriched TF motifs were identified. These included the hypoxia-responsive promoter element (HRPE), transactivated by low-oxygen-stabilized ethylene response group VII (ERFVII) TFs that up-regulate genes key to anaerobic metabolism and flooding survival in Arabidopsis (12-14); a basic helix-loop-helix (bHLH); a MYB; and a WRKY-type motif (Fig. 2D, figs. S15 and S16A, and data S9). At least one of the four motifs was present in $>84 \%$ of the up-regulated SURF genes of rice and $M t$ and $>68 \%$ of those of the Solanum species. HRPE and bHLH motifs predominated near the TSS in all species, with the MYB near the TSS in tomato and WRKY motifs more evenly distributed across the upstream region (fig. S16B). Differential wiring of up-regulated SURFs was evident from the HRPE enrichment in rice (55\%) versus the MYB or bHLH motif enrichment in these three eudicots (fig. S16A and data S9).

Accessibility of chromatin in response to abiotic stress can be rapid and transient $(15,16)$. We hypothesized that concordance between a TF binding site and a THS would be representative of a more static regulatory architecture, whereas discordance could reflect the transient propagation of a stress signal. Chromatin accessibility increased during submergence around HRPE and bHLH sites in rice and $M t$ (fig. S17). A more modest increase was observed for MYB and WRKY sites, potentially representing more rapid and/or transient regulatory interactions (fig. S17). The co-occurrence of an HRPE and THS corresponded with more pronounced polyA RNA up-regulation, with a similar trend observed for bHLH sites in rice and $M t$ (Fig. 2E, fig. S18, and data S10). In $M t$, the presence of a THS alone in the proximal promoter was associated with greater elevation of polyA RNA, and cooccurrence of a MYB and THS corresponded with higher up-regulation than did the presence of the motif alone (Fig. 2F and fig. S18). Repetitive motifs of the same type in accessible regions coincided with greater up-regulation than with repetitive motifs outside THSs. The incidence of multiple HRPE or WRKY motifs corresponded with higher up-regulation in tomato, whereas only an HRPE or multiple bHLH motifs corresponded with up-regulation in $S p$. These results establish a link between the four conserved motifs, chromatin accessibility, and transcriptional activation under submergence.

The discovery of the SURFs and four conserved cis regulatory TF binding motifs in submergenceaccessible chromatin regions motivated us to evaluate whether the conservation prevails in genes maintained at syntenic chromosomal regions 
A

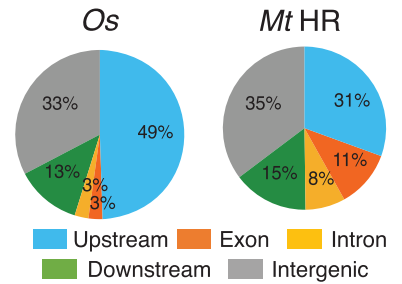

B

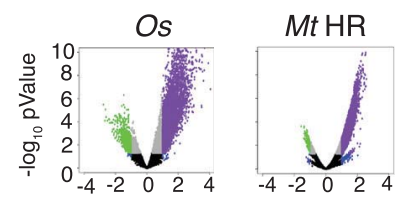

$\log _{2}$ FC THS Sub/Con
C

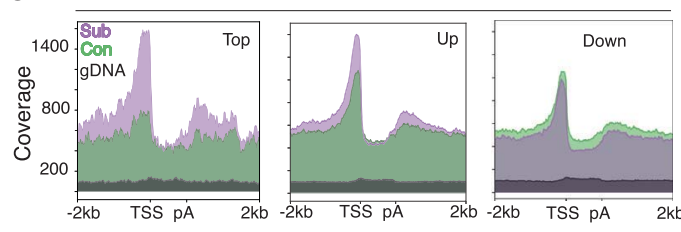

Mt HR

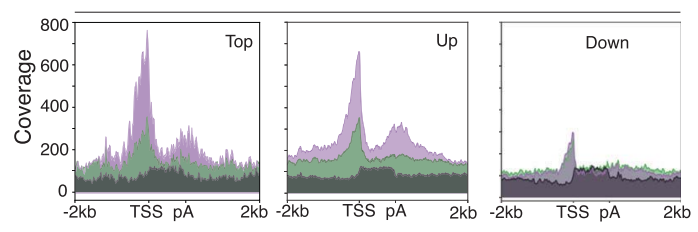

E

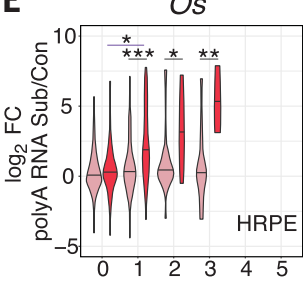

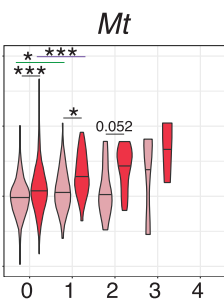

\# HRPE motif in promoter 口Inside THS Outside THS
$\mathbf{F}$

Gene Regulatory Network Building
Motif Discovery and Validation

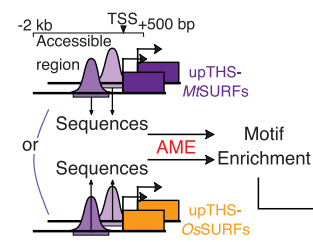

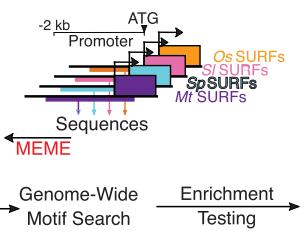

$\rightarrow \begin{aligned} & \text { Genome-Wide } \\ & \text { Motif Search }\end{aligned}$ Testing

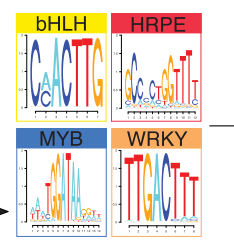

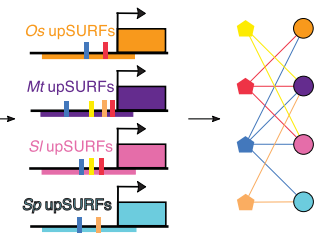

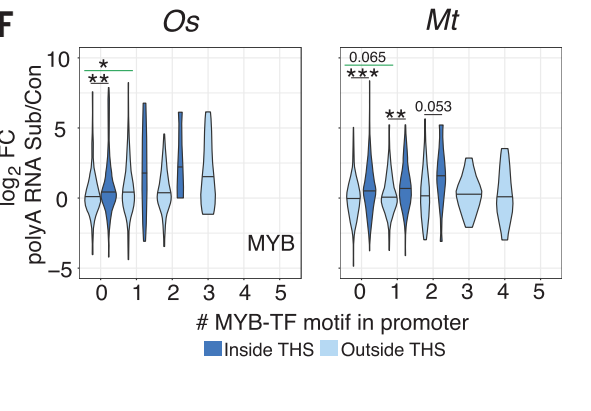

Fig. 2. Enhanced chromatin accessibility and motif enrichment in responsive genes. (A) Accessible chromatin regions (THSs) measured by ATAC-seq. Categories: $2 \mathrm{~kb}$ upstream of the TSS, exons, introns, $1 \mathrm{~kb}$ downstream of pA site, and intergenic. (B) THS change in response to submergence. (C) Control and submergence ATAC-seq reads on genes of up-regulated (Top; cluster 1; Up) and down-regulated (Down) clusters from Fig. 1D. Genomic DNA (gDNA) is ATAC-seq on naked DNA. (D) Discovery pipeline for enriched transcription factor motifs present in up-regulated THSs and SURF promoters, using unsupervised [Multiple Em for Motif Elicitation (MEME)] and supervised [Motif Comparison Tool (TOMTOM), Analysis of Motif Enrichment (AME), and Find Individual Motif Occurrences (FIMO)] methods. (E and $\mathbf{F}$ ) Distribution of $\log _{2} \mathrm{FC}$ polyA RNA submergence and control for SURFs arranged by presence and number of HRPE or MYB motif upstream of the ATG, inside or outside THSs. Student's $t$ test; ${ }^{*} P<0.05$, ${ }^{*} P<0.01$, ${ }^{* *} P<0.001$; values $\leq 0.1$.
Fig. 3. Syntenic genes are more highly expressed.

(A) Median $\log _{2}$ FC of syntenic genes across four species for 12 up-regulated clusters. Dashed lines indicate two clusters with conserved interspecies up-regulation. (B) Plot of $\log _{10}$ reads per kilobase per million reads ( $r p k M)$ for all detected syntenic and nonsyntenic genes under the control condition. Rice synteny was evaluated to Brachypodium distachyon, $\mathrm{Mt}$ to $\mathrm{SI}$, and between Solanum species. Variances between syntenic and nonsyntenic genes are significant in every RNA population ( $F$ test). (C) Control (white columns) and submergence (blue columns) plots for SURF genes. Highly expressed SURF genes under submergence (>50 rpkM) with an HRPE are depicted as a red or blue dot for those located in or outside a THS, respectively. Central horizontal lines indicate median values.
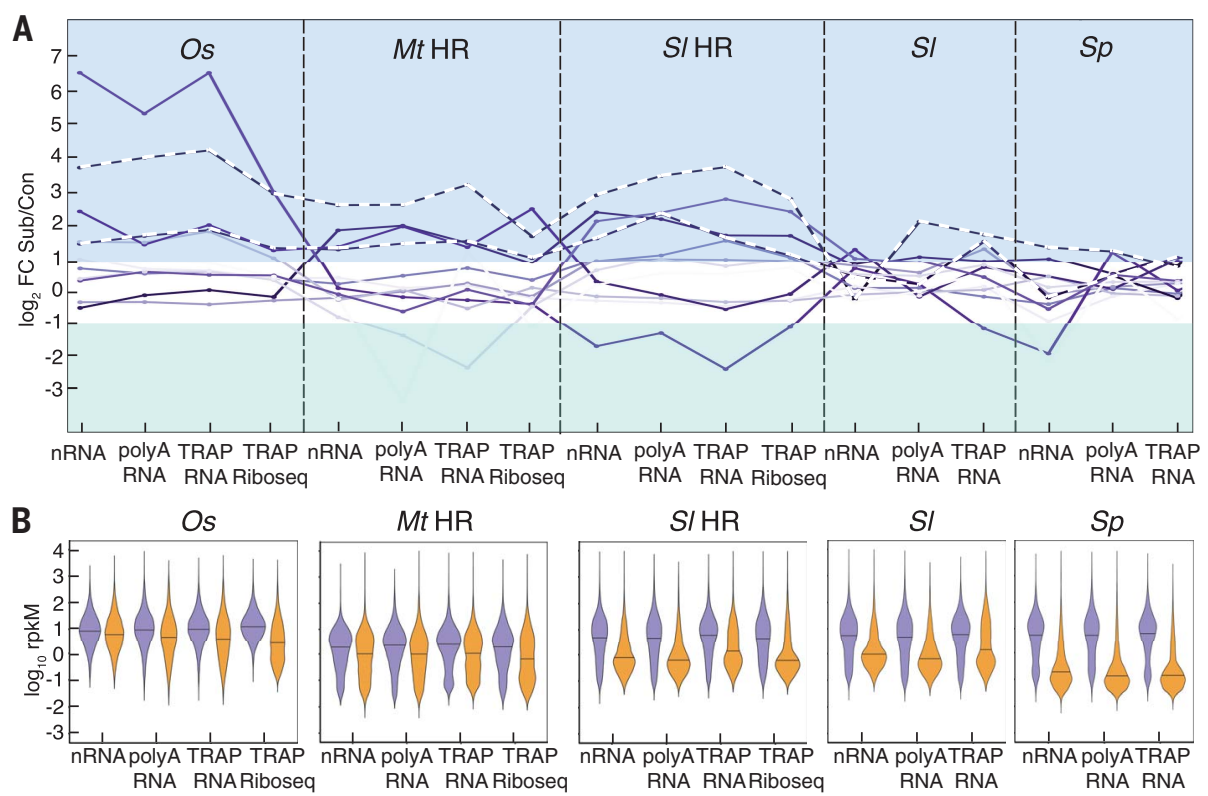

C Os
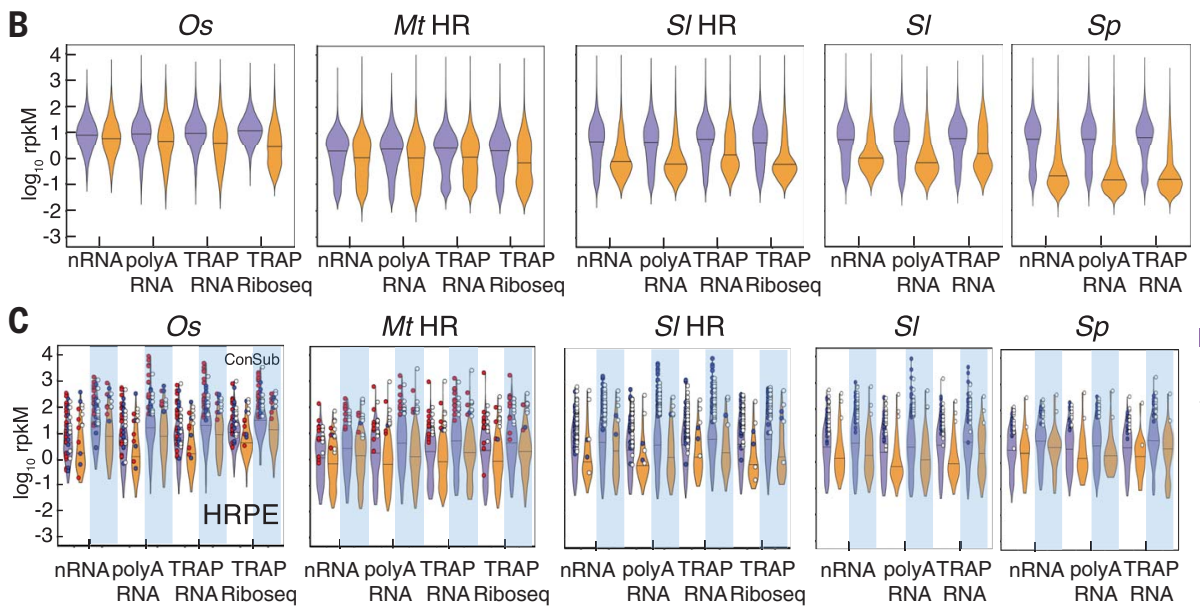

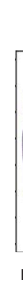

RNA RNARiboseq
nRNA polyATRAP nRNA PolyATRAP RNA RNA

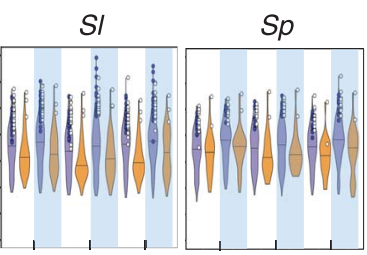


(syntelogs). To do so, the gene activity data were reclustered for the differentially regulated syntelogs across the four species (711), which included 22 of the 68 SURFs (Fig. 3A, fig. S19, and data S11). Syntelog clusters 2 and 3 had coordinated up-regulation across the scales of gene activity in all species. These comprised seven SURFs with functions in anaerobic metabolism, nutrient transport, abscisic acid (ABA) perception, and survival of extreme stress. The up-regulated syntelogs included 32 and 53 SURFs in all three eudicots and the two Solanum species, respectively (figs. S20 to S22 and data S11).

Next, we explored conservation of gene regulation on more recent evolutionary time scales by evaluating the activity of syntelogs of related species (Fig. 3B, fig. S23, and data S12 to S14). Syntenic genes had higher transcript abundance than nonsyntenic genes had, as reported previously (17). This was evident in all RNA populations under both conditions, with the most pronounced difference between syntenic and nonsyntenic genes in the Solanum species. Rice and $M t$ syntenic gene control regions had slightly higher chromatin accessibility than did nonsyntenic genes at the global scale (fig. S14), consistent with their higher expression. Transcript elevation was similar for syntenic and nonsyntenic SURF genes, especially for the Solanum species (Fig. 3C, fig. S24, and data S14), indicating that up-regulated nonsyntenic genes have maintained or acquired features enabling their stress activation. Consistent with this, most highly expressed syntenic and nonsyntenic SURF genes contained at least one of the four TF motifs recognized (80\% rice, $80 \% \mathrm{Mt}$, $>70 \%$ Solanum species) (Fig. 3C, fig. S24, and data S15). Most TF motifs were coincident with THSs in rice and $M t$. Although the number of highly expressed but nonsyntenic SURF genes was fewer than six in the Solanum species, all from $\mathrm{Sl}$ contained at least one motif. The four identified TF motifs are therefore a broadly conserved feature of both syntenic and nonsyntenic submergenceresponsive genes.

To appraise conservation in regulation across eudicots and monocots, we built networks that associate TF motif presence with each up-regulated SURF gene for each species (Fig. 4A, figs. S25 to $\mathrm{S} 28$, and data S16). The individual species networks emphasize the presence of species-specific motif biases. The combinatorial nature of target gene regulation was also evident (overlapping outer circles of network), with $>70 \%$ of the genes having more than one of the four motifs. Syntenic up-regulated SURF genes across the four species (represented with black borders) expose a single conserved putative regulatory network (Fig. 4B, fig. S29, and data S16). This network illustrates conservation of TF motifs of syntelogs of responsive genes, in addition to the HRPE regulated by ERFVIIs.

As oxygen levels decline below a threshold, constitutively synthesized ERFVIIs accumulate because of attenuation of their conversion into an $\mathrm{N}$-degron for active turnover (1). The unified SURF network uncovered HRPE conservation across eudicots-monocots in promoters of genes essential to anaerobic metabolism and hypoxia survival, including PLANT CYSTEINE OXIDASE (PCO) genes (Fig. 4C, fig. S30, and data S17), which catalyze the oxygen-promoted degradation of ERFVIIs to temper the adaptive response (18). The up-regulated SURF genes included ERFVIIs in all four species, with at least one with an HRPE motif, suggesting possible autoregulation (fig. S31).

The syntelog network also identified conservation of bHLH motif enrichment in genes not well associated with submergence [i.e., PYRABACTIN RESISTANCE 1/PYRI-LIKE (PYL)] (Fig. 4B and fig. S32) and MYB motif enrichment in genes that contribute to hypoxia tolerance (14) (fig. S33 and S34). The up-regulation of these genes often coincided with a TF motif in a region of submergence-enhanced chromatin accessibility (Fig. 4, D to G, and fig. S33), supporting functionality of the regulatory sequences. As for the ERFVIIs, the up-regulated SURF genes included bHLH, MYB, and WRKY family members (fig. S31).
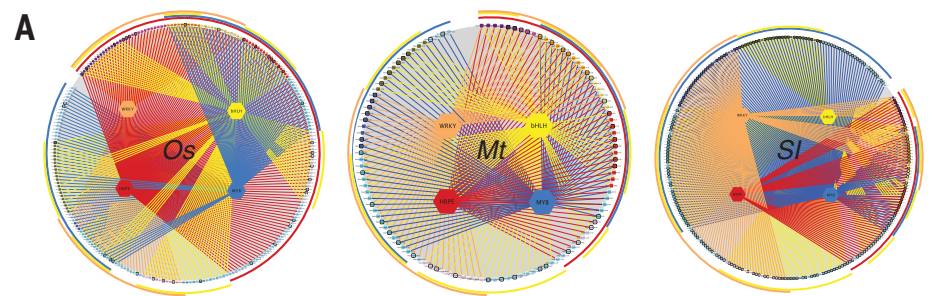

B

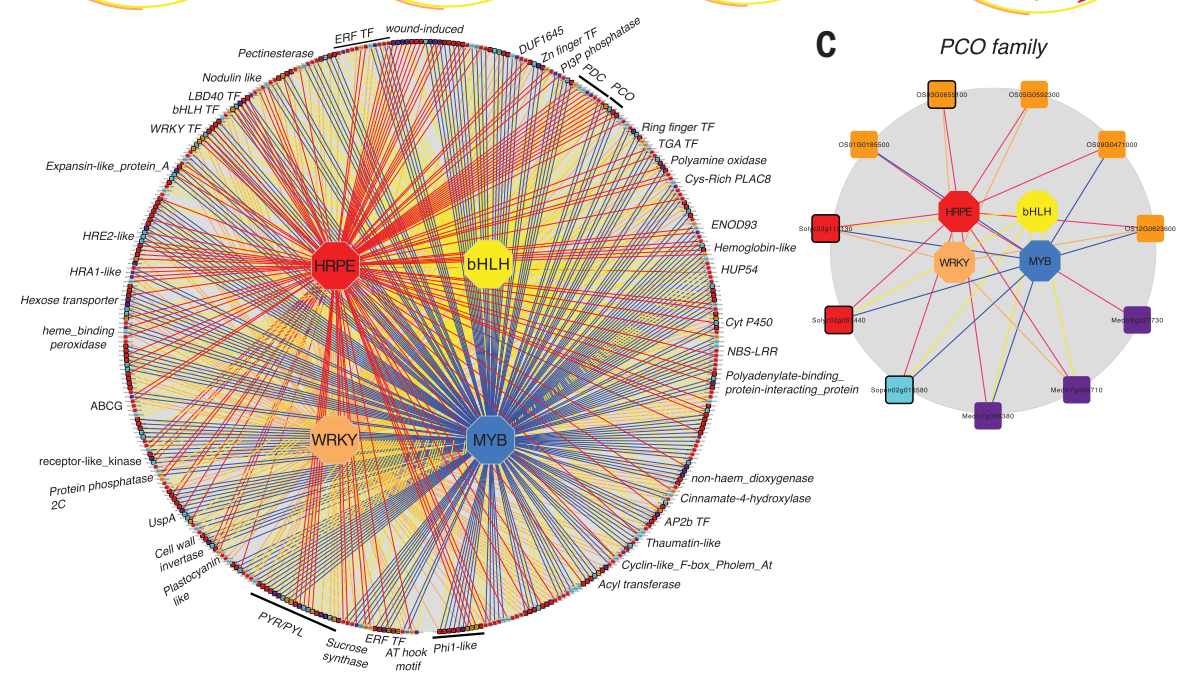

D
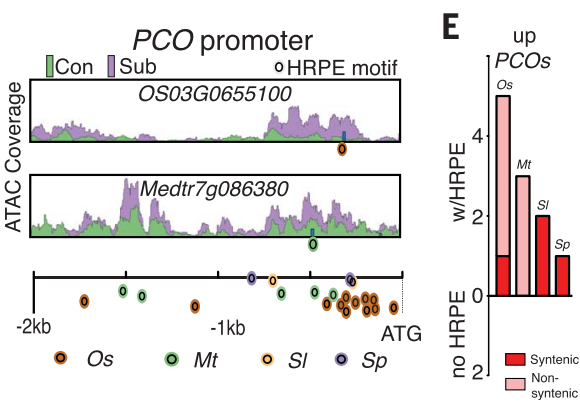

$\mathbf{F}$
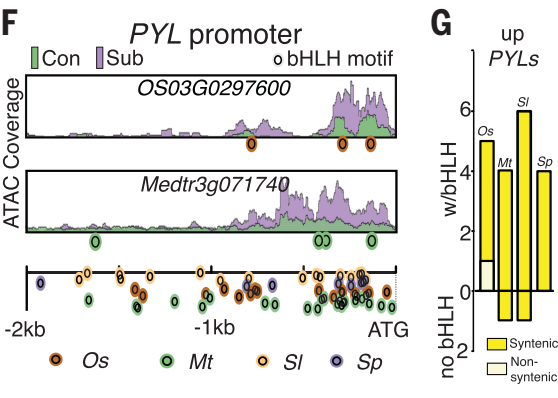

Fig. 4. Conserved transcription factor motifs in SURFs and accompanying chromatin dynamics. (A) Regulatory networks for up-regulated SURF genes (expanded in figs. S25 to S28). Hexagons, TFs; rectangles, genes; colored lines (edges), interactions of promoter and TF based on motif presence. Outer circles: genes grouped with shared motifs. Genes with black borders have a syntenic ortholog (rice to $M t$; Mt to SI; and between Solanum species). (B) Network for syntenic conserved SURF genes across species (expanded in fig. S29). Genes of alternating families have alternating gray or black borders. Families represented in three species are labeled. ABCG, ATP-binding cassette G; Cyt, cytochrome; LRR, leucine-rich repeat. (C) Regulatory network of $P C O$ up-regulated genes. Syntenic orthologs have black borders. ( $\mathbf{D}$ and $\mathbf{F}$ ) Chromatin accessibility in promoters of syntenic PCO and PYL. ATAC coverage scale is the same for genes shown in each panel. (Bottom) Locations of HRPE or bHLH motifs for four species. ( $E$ and $\mathbf{G}$ ) Number of up-regulated genes containing motifs classified by syntenic and nonsyntenic. 
Information from single genes is used in breeding or modifying crops for stress tolerance. The use of multiscale gene regulatory information of gene families across flowering plant clades to infer regulatory networks demonstrates that conservation of flooding resilience mechanisms is complex and involves diverse regulatory mechanisms. Targeted manipulation of the four submergenceactivated modules and seven SURF loci discovered in this study with the greatest interspecies conservation might be used to enhance flooding tolerance of susceptible crops.

\section{REFERENCES AND NOTES}

1. L. A. C. J. Voesenek, J. Bailey-Serres, New Phytol. 206, 57-73 (2015).

2. C. Branco-Price, K. A. Kaiser, C. J. H. Jang, C. K. Larive, J. Bailey-Serres, Plant J. 56, 743-755 (2008).

3. R. Sorenson, J. Bailey-Serres, Proc. Natl. Acad. Sci. U.S.A. 111 2373-2378 (2014).

4. P. Juntawong, T. Girke, J. Bazin, J. Bailey-Serres, Proc. Natl. Acad. Sci. U.S.A. 111, E203-E212 (2014)

5. R. B. Deal, S. Henikoff, Nat. Protoc. 6, 56-68 (2011)

6. A. Mustroph et al., Proc. Natl. Acad. Sci. U.S.A. 106, 18843-18848 (2009).
7. J. D. Buenrostro, P. G. Giresi, L. C. Zaba, H. Y. Chang, W. J. Greenleaf, Nat. Methods 10, 1213-1218 (2013)

8. N. T. Ingolia, S. Ghaemmaghami, J. R. S. Newman, J. S. Weissman, Science 324, 218-223 (2009).

9. D. M. Goodstein et al., Nucleic Acids Res. 40, D1178-D1186 (2012).

10. M. Klecker et al., Plant Physiol. 165, 774-790 (2014).

11. K. A. Maher et al., Plant Cell 30, 15-36 (2018).

12. P. Gasch et al., Plant Cell 28, 160-180 (2016).

13. S. C. Lee et al., New Phytol. 190, 457-471 (2011).

14. A. Mustroph et al., Plant Physiol. 152, 1484-1500 (2010).

15. B. O. R. Bargmann et al., Mol. Plant 6, 978-980 (2013).

16. A. Para et al., Proc. Natl. Acad. Sci. U.S.A. 111, 10371-10376 (2014)

17. J. W. Walley et al., Science 353, 814-818 (2016).

18. D. A. Weits et al., Nat. Commun. 5, 3425 (2014).

19. J. Barba-Montoya, M. Dos Reis, H. Schneider,

P. C. J. Donoghue, Z. Yang, New Phytol. 218, 819-834 (2018).

\section{ACKNOWLEDGMENTS}

We thank members of our labs, R. Mataki, S. Cabanlit, E. Viox, K. Tran, A. Addetia, S. Winte, M. Hummel, T. Lee, A. Mason, and H. Nakayama for support and discussions and J. Bazin, D. Koenig, D. Kliebenstein, T. Bailey, A. Reynoso, M. Covington, and S. Gray for guidance. Funding: Supported by United
States National Science Foundation Plant Genome Research Program (IOS-1238243) to R.B.D., N.S., S.M.B., and J.B.-S. a Finnish Cultural Foundation fellowship to K.K., and an HHMI Faculty Scholar Fellowship to S.M.B. National Science Foundation (IOS-1558900) to N.S. Author contributions: M.A.R., K.K., M.B., G.P., D.A.W., N.S., S.M.B., R.B.D., and J.B.-S. conceived the study, designed experiments, and performed analysis; M.A.R., K.K., M.B., G.P., D.A.W., A.Y., K.H., K.Z., and M.W. performed experiments. M.A.R., K.K., M.B., N.S., S.M.B., R.B.D., and J.B.-S. wrote the manuscript. J.B.-S. can be contacted for rice genetic materials, R.B.D. for Medicago genetics materials, S.M.B. for tomato genetic materials, and N.S. for S. pennellii genetic materials. Competing interests: The authors declare no competing interests. Data and material availability: Sequence data are deposited in GEO (accession GSE128680); code and resources are in http://plant-plasticity.github.io/ data-and-code/. All other data are in the main paper or supplementary materials.

\section{SUPPLEMENTARY MATERIALS}

science.sciencemag.org/content/365/6459/1291/suppl/DC1 Materials and Methods

Figures S1 to S34

References (20-72)

Data S1 to S18

1 May 2019; accepted 26 August 2019

10.1126/science.aax8862 


\section{Science}

\section{Evolutionary flexibility in flooding response circuitry in angiosperms}

Mauricio A. Reynoso, Kaisa Kajala, Marko Bajic, Donnelly A. West, Germain Pauluzzi, Andrew I. Yao, Kathryn Hatch, Kristina Zumstein, Margaret Woodhouse, Joel Rodriguez-Medina, Neelima Sinha, Siobhan M. Brady, Roger B. Deal and Julia Bailey-Serres

Science 365 (6459), 1291-1295.

DOI: $10.1126 /$ science.aax8862

\section{Flood-resistance from gene regulation}

Some plants tolerate flooding better than others. Reynoso et al. compared gene regulatory networks activated by flooding in rice, which is adapted to flooding, with those in species less adapted to flooding. Flood-related gene regulation was characterized according to chromatin accessibility as well as transcription. Although flood response circuitry is evident in dryland species as well, its activation is greater in wetland rice.

Science, this issue p. 1291

ARTICLE TOOLS

SUPPLEMENTARY MATERIALS

REFERENCES

PERMISSIONS http://science.sciencemag.org/content/365/6459/1291

http://science.sciencemag.org/content/suppl/2019/09/18/365.6459.1291.DC1

This article cites 70 articles, 19 of which you can access for free http://science.sciencemag.org/content/365/6459/1291\#BIBL

http://www.sciencemag.org/help/reprints-and-permissions

Use of this article is subject to the Terms of Service

Science (print ISSN 0036-8075; online ISSN 1095-9203) is published by the American Association for the Advancement of Science, 1200 New York Avenue NW, Washington, DC 20005. The title Science is a registered trademark of AAAS.

Copyright (C) 2019 The Authors, some rights reserved; exclusive licensee American Association for the Advancement of Science. No claim to original U.S. Government Works 